\title{
ON A PROBLEM OF WALSH CONCERNING THE HADAMARD THREE CIRCLES THEOREM
}

\author{
BY \\ MAURICE H. HEINS
}

1. Introduction. The following problem was proposed to the author by Professor (now Lieutenant Commander) Walsh:

Let $\mathfrak{A}$ denote the class of functions $f(z)$ satisfying the following requirements: I. $f(z)$ is analytic for $|z|<R(>0)$; II. $|f(z)|<M(>0)$ for $|z|<R$; III. $|f(z)|$ $\leqq m(<M)$ for $|z| \leqq r(<R)$. Under these circumstances it is required to determine

$$
\underset{s \in \mathfrak{R}}{\text { l.u.b. }} M(f, \rho)
$$

where $r<\rho<R$ and $M(f, \rho)=\max _{|z|=\rho}|f(z)|$, and the associated extremal functions of the class $\mathfrak{A}$.

The requirements of this problem specialize the hypotheses of the Three Circles Theorem in two directions. First, since the functions $f(z)$ of class $\mathscr{A}$ are analytic for $|z|<R$, they are single-valued; the Three Circles Theorem admits to competition functions whose moduli are single-valued, but which need not themselves be single-valued. Second, the functions of class $\mathfrak{A}$ are analytic for $|z|<R$, whereas the functions admitted in the Hadamard theorem are required to be analytic only for $r<|z|<R$. Since the extremal functions of the Three Circles Theorem are analytic throughout $|z|<R$ only when $m / M$ is a positive integral power of $r / R$, the appraisal given by this theorem for $M(f, \rho)$ with $f \in \mathfrak{A}$ is the best possible only under very restrictive hypotheses on $m, M, r, R$. By hypotheses II and III on $f \in \mathfrak{A}$, the class $\mathfrak{A}$ is compact and hence there certainly exists a function $f_{0}(z)$ of this class for which

$$
M\left(f_{0}, \rho\right)=\text { l.u.b. }_{f \in \mathscr{I}} M(f, \rho) .
$$

The principal object of the present paper is to study the descriptive properties of these extremal functions. The method of approach is typically Tchebycheffian in spirit $[1,13]\left({ }^{1}\right)$; but there is a fundamental difference between the present problem and a characteristically Tchebycheffian problem. In the latter, one is concerned with determining an extremal function in a class of functions where the concept of degree is introduced a priori (for example, algebraic polynomials, trigonometric polynomials, rational func-

Presented to the Society, September 13, 1943; received by the editors April 1, 1943, and, in revised form, October 7, 1943.

(1) Numbers in brackets refer to the bibliography at the end of the paper. 
tions) and a given bound is assigned for the degree of all competing functions. In the present problem no such restriction is imposed. It will be seen that the extremal functions are rational, defining $(1, k)$ conformal mappings of $|z|<R$ onto $|w|<M$, where $k$ is a positive whole number, and it will be one of our objects to show how $k$ may be determined in terms of the data of $\mathfrak{A}$.

In addition to considering the problem for $\mathfrak{A}$, related extremal problems will be studied in the course of the present paper.

The following simplifying assumptions may be made without loss of generality: $(\alpha) M=1,(\beta) R=1,(\gamma) f(\rho)=M(f, \rho)$. It will be assumed that these normalizations hold throughout the discussion.

Mention should be made of related investigations. The corresponding extremal problem for the class of functions which satisfy in addition to the hypotheses of the Three Circles Theorem the requirement that they be singlevalued in the annulus of definition has been treated by Carlson [4], Teichmüller [12]; and the author [5]. The related problem for the class of functions satisfying the requirements of $\mathfrak{A}$ with III replaced by the condition that $|f(z)| \leqq m(<1)$ for $z \in \gamma$ (or by closely related conditions), where $\gamma$ is a Jordan arc with one end point deleted, $\gamma$ lying in $|z|<1$, the deleted end point being on $|z|=1$, has been studied by E. Schmidt [10], R. Nevanlinna [2], and Beurling [2].

2. Properties of the extremal function. It has already been remarked that by virtue of hypotheses II and III there exists an extremal function $f_{0}(z) \in \mathfrak{A}$. Before showing that the extremal function is unique and defines a $(1, k)(k$ a positive integer) conformal mapping $\left({ }^{2}\right)$ of the interior of the unit circle onto itself, it should be noted that two simple sets of cases can be handled quite directly. If $m=r^{n}(n=1,2, \cdots)$, then it follows from the Three Circles Theorem that the extremal function is unique and is precisely $z^{n}$. If $r<m<1$, the extremal function and the corresponding extremal value may be readily obtained. Let $\mu$ denote l.u.b. $f \in \mathfrak{x} f(\rho)$ and let $f_{0}(z)$ denote a corresponding extremal function. Clearly, $\left[f_{0}(z)+\overline{f_{0}(\tilde{z})}\right] / 2$ is also an extremal function and in addition is real for $z$ real. Now let $\mu^{*}$ denote the largest possible positive value which a function $\phi(z)$ can attain at $z=\rho$, where $\phi(z)$ not only is analytic and of modulus less than unity for $|z|<1$, but also is real for $z$ real and satisfies $|\phi(r)| \leqq m$. By Pick's formulation of Schwarz's lemma [3], it follows that

$$
\frac{\mu^{*}-m}{1-m \mu^{*}}=\frac{\rho-r}{1-\rho r},
$$

and that the unique extremal function, $\phi_{0}(z)$, is defined by

$$
\frac{\phi_{0}(z)-m}{1-m \phi_{0}(z)}=\frac{z-r}{1-r z} .
$$

(2) For the theory of such mappings see [6] where reference is made to the pertinent literature. 
Note that $\mu \leqq \mu^{*}$. Since $r<m<1$, it is readily deduced that

$$
\left|\phi_{0}(z)\right| \leqq m \text { for }|z| \leqq r .
$$

It follows from (2.3) that $\phi_{0}(z)$ is a member of $\mathfrak{A}$ and hence extremal and that $\mu=\mu^{*}$. Further, $\phi_{0}(z)$ is unique. If $\Phi(z)$ were an extremal function distinct from $\phi_{0}(z)$, then $\Phi(r) \neq m$; otherwise $\Phi(z)$ would have to coincide with $\phi_{0}(z)$, since by Pick's theorem $\phi_{0}(z)$ is the only function analytic and of modulus less than unity for $|z|<1$ which satisfies the interpolation requirements: $\phi_{0}(r)=m$, $\phi_{0}(\rho)=\mu^{*}$. But if $\Phi(r) \neq m$, then $\psi(z) \equiv[\Phi(z)+\overline{\Phi(\bar{z})}] / 2$ is also extremal, real for $\%$ real, and $\psi(r)<m$. By Pick's theorem

$$
\frac{\mu^{*}-\psi(r)}{1-\mu^{*} \psi(r)} \leqq \frac{\rho-r}{1-\rho^{r}}
$$

which is manifestly impossible if $(2.1)$ obtains and $\psi(r)<m$. Hence $\phi_{0}(z)$ is unique.

If $m<r$, then it is readily verified that (2.1) and (2.2) do not yield the extremal value and the corresponding extremal function, since $\left|\phi_{0}(-r)\right|$ would then exceed $m$ and hence $\phi_{0}(z)$ would not belong to $\mathfrak{A}$.

To return to the general situation, it suffices now to treat the problem for only those $m$ which are less than $r$ and are not positive integral powers of $r$. Under these circumstances let $f_{0}(z)$ be an extremal function. Then $M\left(f_{0}, r\right)=m$. If this were not the case and $M\left(f_{0}, r\right)<m$, then one could consider the function

$$
T_{\lambda}\left[f_{0}(z)\right],
$$

where $T_{\lambda}(Z)$ is defined by

$$
\frac{T_{\lambda}-1}{T_{\lambda}+1}=\lambda \frac{Z-1}{Z+1}
$$

If $\lambda$ is sufficiently near one, then certainly

$$
M\left\{T_{\lambda}\left[f_{0}(z)\right], r\right\}<m
$$

and hence $T_{\lambda}\left[f_{0}(z)\right] \in \mathfrak{A}$. On the other hand, $T_{\lambda}\left[f_{0}(\rho)\right]>f_{0}(\rho)$ and this implies that $f_{0}(z)$ is not extremal. The assertion that $M\left(f_{0}, r\right)=m$ follows.

It is conceivable that for some extremal function $f_{0}(z),\left|f_{0}(z)\right|=m$ for an infinite set of distinct $z$ on the circle $|z|=r$. If this were so,

$$
\left|f_{0}\left(r e^{i \theta}\right)\right| \underset{\theta}{\equiv} m
$$

It will now be shown that the relation $(2.8)$ is untenable. Contrariwise, $f_{0}(z)$ would be a rational function which would define a $(1, k)$ conformal mapping of $|z|<r$ onto $|w|<m[6$, p. 55]. Since $m$ is assumed to be not a positive integral power of $r,\left|f_{0}(z)\right|$ would attain the value unity at most a finite num- 
ber of times for $|z|=1$. Two cases are to be distinguished according as $\left|f_{0}(1)\right|$ $=1$ or not.

To reach a contradiction an auxiliary function is introduced in the following manner. Let $\alpha$ denote the arc of the unit circle defined by

$$
\alpha:|\theta|<\theta_{0}<\pi
$$

$\left(\theta_{0}\right.$ positive $)$

and let $u(z ; \alpha)$ denote the unique function which is harmonic and bounded for $|z|<1$ and which attains the boundary value one on $\alpha$ and the boundary value zero on the arc of $|z|=1$ complementary to $\alpha$. From a study of the level curves of $u(z ; \alpha)$ (which are the arcs of circles in $|z|<1$ with end points at $e^{i \theta_{0}}$ and $\left.e^{-i \theta_{0}(3)}\right)$, it follows that

$$
u(\rho ; \alpha)>\max _{|z|=r} u(z ; \alpha) .
$$

Now let $\alpha^{*}$ denote the subarc of $\alpha$ defined by

$$
\alpha^{*}:|\theta|<\Theta_{0}<\theta_{0}
$$

and let $u\left(z ; \alpha^{*}\right)$ have the same connotation relative to $\alpha^{*}$ that $u(z ; \alpha)$ has relative to $\alpha$. If $\Theta_{0}$ is chosen sufficiently small, then $\max _{|z| \leq \rho} u\left(z ; \alpha^{*}\right)$ may be made arbitrarily small and hence

$$
u(\rho ; \alpha)-u\left(\rho ; \alpha^{*}\right)>\max _{|z|=r}\left\{u(z ; \alpha)-u\left(z ; \alpha^{*}\right)\right\} .
$$

These preliminaries agreed upon, if $\left|f_{0}(1)\right|<1$, let $\theta_{0}$ be chosen so small that $\max _{|\theta| \geqq \theta_{0}}\left|f_{0}\left(e^{i \theta}\right)\right|<1$, and let $U(z)$ denote $u(z ; \alpha)$. If $\left|f_{0}(1)\right|=1$, then let $\theta_{0}$ be chosen so small that $\left|f\left(e^{i \theta}\right)\right|=1$ for $|\theta| \leqq \theta_{0}$ implies $\theta=0$, and let $\Theta_{0}$ be chosen so small that the relation (2.12) prevails; in this case let $U(z)$ denote $u(z ; \alpha)-u\left(z ; \alpha^{*}\right)$. Now let $\epsilon$ be real and such that in the first case $\left(\left|f_{0}(1)\right|<1\right)$

$$
e^{-\epsilon}=\max _{|\theta| \leqq \theta_{0}}\left|f_{0}\left(e^{i \theta}\right)\right|,
$$

and in the second case $\left(\left|f_{0}(1)\right|=1\right)$

$$
e^{-\epsilon}=\max _{\Theta_{0} \leqq|\theta| \leqq \theta_{0}}\left|f_{0}\left(e^{i \theta}\right)\right| .
$$

In either case let $V(z)$ denote the unique conjugate of $U(z)$ which is so normalized that $V(0)=0$. Finally, let $w(z)$ denote

$$
\exp \{\epsilon[U(z)+i V(z)]\} \text {. }
$$

With this choice of $w(z)$, the function $w(z) f_{0}(z)$ is clearly analytic and of modulus less than unity for $|z|<1$. It does not belong, however, to $\mathfrak{A}$. But the function $w(z) f_{0}(z) /\left\{\max _{|z|=r}|w(z)|\right\}$ does belong to $\mathfrak{A}$. Alternatively from (2.10) or (2.12)

(3) Details are to be found in chap. 1 of [7]. 


$$
\frac{w(\rho) f_{0}(\rho)}{\max _{|z|=r}|w(z)|}>f_{0}(\rho)
$$

and clearly $f_{0}(z)$ cannot be extremal. The following theorem is thus established.

THEOREM 2.1. If $m$ is not a positive integral power of $r$, and if $f_{0}(z)$ is an extremal function of $\mathfrak{A}$, then $M\left(f_{0}, r\right)=m$ and the relation $\left|f_{0}(z)\right|=m$ is satisfied for only a finite number of distinct $z$ satisfying $|z|=r$.

From the typical Tchebycheffian point of view it is now natural to place emphasis on the finite set of points of $|z|=r$ where $\left|f_{0}\right|=m$. This assumption is justified by the following theorem.

THEOREM 2.2. For any $m(0<m<1)$ the extremal function $f_{0}(z)$ is unique. The function $f_{0}(z)$ is rational, real for $z$ real, and defines $a(1, k)$ conformal map of $|z|<1$ onto itself.

Recall the following results from the Pick-Nevanlinna theory of interpolation [14, chap. 10]: Let $z_{1}, z_{2}, \cdots, z_{n}$ be $n$ distinct points of $|z|<1$ and let $w_{1}, w_{2}, \cdots, w_{n}$ be $n$ points of $|w|<1$. If there exists a function $f(z)$ which is analytic and of modulus less than unity for $|z|<1$ such that $f\left(z_{\lambda}\right)=w_{\lambda}$ $(\lambda=1,2, \cdots, n)$, then it is either unique and in this case $f(z)$ defines a $(1, k)$ conformal mapping of $|z|<1$ onto $|w|<1, k$ being at most $n-1$, or else there is an infinite set of functions satisfying these requirements. If this is so, and if $\zeta$ is a point of $|z|<1$ distinct from all the $z_{\lambda}$, then the set of possible values for $f(\zeta)$ fill a closed circle $K_{\zeta}$, lying in the interior of $|w|<1$. Further, if the additional requirement that $f(\zeta)$ shall be a given point of the circumference of $K_{\zeta}$ is imposed, then the function $f(z)$ so restricted is unique.

To return to the question under consideration, let $f_{0}(z)$ be an alleged extremal function of $\mathfrak{A}$, and let $z_{1}, z_{2}, \cdots, z_{n}$ denote the finite set of points on $|z|=r$ for which $\left|f_{0}\left(z_{\lambda}\right)\right|=m(\lambda=1,2, \cdots, n)$, and let $w_{\lambda}$ denote $f_{0}\left(z_{\lambda}\right)$ for $\lambda=1,2, \cdots, n$, and finally, as above, let $\mu=f_{0}(\rho)$. It follows from the PickNevanlinna theory that either there exists a unique function $\phi(z)$, which is analytic and of modulus less than unity for $|z|<1$, satisfying

$$
\phi\left(z_{\lambda}\right)=w_{\lambda}(\lambda=1,2, \cdots, n) ; \phi(\rho)=\mu,
$$

or else the class of functions $\phi(z)$ satisfying these requirements contains more than a single member. In the first case, $f_{0}(z)$ is necessarily identical with $\phi(z)$ and hence defines a $(1, k)$ conformal map of at most degree $n$ of $|z|<1$ onto itself. In the second case, certainly the class of functions $\Phi(z)$, analytic and of modulus less than unity for $|z|<1$, which satisfy

$$
\Phi\left(z_{\lambda}\right)=w_{\lambda} \quad(\lambda=1,2, \cdots, n)
$$


contains the class $\{\phi(z)\}$. Let $K_{\rho}$ denote the circle in $|w|<1$ which the set $\{\Phi(\rho)\}$ fills. It is clear that $\mu$ is an interior point of $K_{p}$; otherwise the function $\phi(z)$ satisfying (2.13) would be unique. Now let $\mu^{*}$ denote the unique point of the positive real axis which lies on the circumference of $K_{\rho}$ and is farthest from the origin of the $w$-plane, and let $\Phi_{0}(z)$ denote the unique member of $\{\Phi(z)\}$ for which $\Phi_{0}(\rho)=\mu^{*}$. Since $\mu^{*}>\mu$, the function

$$
\psi(z ; \tau) \equiv \tau f_{0}(z)+(1-\tau)\left(\left(\mu+\mu^{*}\right) / 2 \mu^{*}\right) \Phi_{0}(z)
$$$$
(0<\tau<1)
$$

has the property that for this set of $\tau, \psi(\rho ; \tau)>f_{0}(\rho)$. Observe that the following relations hold:

$$
\left|\left(\left(\mu+\mu^{*}\right) / 2 \mu^{*}\right) \Phi_{0}\left(z_{\lambda}\right)\right|<\left|w_{\lambda}\right| \quad(\lambda=1,2, \cdots, n) .
$$

Hence for $\tau$ sufficiently near one, $M[\psi(z ; \tau), r]<m$. For such $\tau, \psi(z ; \tau) \in \mathfrak{A}$ and yet $\psi(\rho ; \tau)>\mu$. The contradiction is manifest. It follows that every extremal function is rational and defines a $(1, k)$ conformal map of $|z|<1$ onto itself.

To establish uniqueness, recall that any $(1, k)$ conformal map $r(z)$, of $|z|<1$ onto itself, is such that $|z|=1$ implies $|r(z)|=1$. Suppose that $f_{0}(z)$ and $F_{0}(z)$ are two distinct extremal functions. Then so is $\left[f_{0}(z)+F_{0}(z)\right] / 2$. But

$$
\left|\left[f_{0}(z)+F_{0}(z)\right] / 2\right|=1 \text { for }|z|=1
$$

implies that $f_{0}(z) \equiv F_{0}(z)$.

To show that $f_{0}(z)$, extremal, is real for $z$ real, observe that $\left[f_{0}(z)+\overline{f_{0}(\bar{z})}\right] / 2$ is extremal and real for $z$ real.

Theorem 2.2 follows.

3. Dependence of $\mu$ and the extremal function upon the data of the problem. In this section certain elementary results concerning the dependence of $\mu$ and the extremal function upon the data of the problem are established. These results will be useful in determining the degree of the extremal function.

First, for fixed $r$ and $\rho, \mu$ is a continuous strictly increasing function of $m$ for $0<m<1$, the image of $(0<m<1)$ with respect to $\mu(m)$ being the interval $(0<\mu<1)$. It is trivial that $m_{1}<m_{2}$ implies $\mu\left(m_{1}\right) \leqq \mu\left(m_{2}\right)$. On the other hand, it is impossible that for $m_{1}<m_{2}, \mu\left(m_{1}\right)=\mu\left(m_{2}\right)$. For then the extremal function $\phi_{1}(z)$, with data $\left(r, \rho, m_{1}\right)$, would belong to the class of functions $\{f\}$ of that $\mathfrak{A}$ for which $M(f, r) \leqq m_{2}$. Let $\phi_{2}(z)$ denote the extremal function for this class. By Theorem 2.2, $\phi_{1} \equiv \phi_{2}$, and from Theorem 2.1, $M\left(\phi_{1}, r\right)=m_{2}$. The contradiction is manifest. To establish the continuity of $\mu(m)$, let $m_{0}$ be a number strictly between zero and one. It is clear that for $0<m<m_{0}, \lim _{m \rightarrow m_{0}} \mu(m)$ $=\mu\left(m_{0}\right)$. This follows from the observation that for $m$ satisfying $(1-\epsilon) m_{0}$ $<m<m_{0}$, where $\epsilon$ is positive and near zero,

$$
(1-\epsilon) \mu\left(m_{0}\right)<\mu\left[(1-\epsilon) m_{0}\right]<\mu(m)<\mu\left(m_{0}\right) .
$$

On the other hand, if, for $m>m_{0}, \mu^{*}$ denotes $\lim _{m \rightarrow m_{0}} \mu(m), \mu^{*}=\mu\left(m_{0}\right)$. If this 
were not the case and $\mu^{*}>\mu\left(m_{0}\right)$, let $f_{0}(z ; m)$ denote the extremal function for the class $\{f\}$ with $M(f, r) \leqq m$, and let $\left\{m_{k}\right\}$ denote a monotone strictly decreasing sequence with limit $m_{0}$ such that $\left\{f_{0}\left(z ; m_{k}\right)\right\}$ converges continuously in the sense of Carathéodory for $|z|<1$ as $k \rightarrow \infty$. Let $f^{*}(z)$ denote the limit function. Now $M\left(f^{*}, r\right) \leqq m_{0}$ and $f^{*}(\rho)=\mu^{*}>\mu\left(m_{0}\right)$; this is impossible.

The fact that the image of $(0<m<1)$ with respect to $\mu(m)$ is $(0<\mu<1)$ is immediate.

A corollary of this result is that, if $f_{0}(z ; m)$ denotes the extremal function for the class $\{f\}$ with $M(f, r) \leqq m$, then $\lim _{m \rightarrow m_{0}} f_{0}(z ; m)=f_{0}\left(z ; m_{0}\right)$ continuously in the sense of Caratheodory for $|z|<1$.

$A$ dual extremal problem. Let the positive numbers $r, \rho, \mu$ be given, all less than one, and let $r<\rho$. Further let $\mathfrak{B}_{\mu}$ denote the class of functions $\phi(z)$, analytic and of modulus less than one for $|z|<1$, which satisfy $\phi(\rho)=\mu$. It is required to determine

$$
\underset{\phi \in \mathfrak{P}_{\mu}}{\text { g.l.b. }} M(\phi, r)
$$

and the associated extremal functions.

This problem is dual to the problem originally proposed for $\mathfrak{A}$. As a matter of fact, the extremal function for that $\mathfrak{A}$ with the data $(r, \rho, m)$ is the same as the extremal function for that $\mathfrak{B}_{\mu}$ with the data $(r, \rho, \mu(m))$. To see this let $\phi_{0}(z ; \mu)$ denote an extremal function of $\mathfrak{B}_{\mu}$ with $\mu=\mu(m)$. Then $M\left(\phi_{0}, r\right)$ $=$ g.l.b. ${ }_{\phi \in \mathfrak{B}_{\mu}} M(\phi, r) \leqq m$. On the other hand, $\phi_{0}$ belonging to the $\mathfrak{A}$ class $\{f\}$ with $M(f, r) \leqq m$, it is impossible that $M\left(\phi_{0}, r\right)<m$. The assertion follows. The substitution of the $\mathfrak{B}$-problem for the $\mathfrak{A}$-problem will be of great service.

It should be remarked that, since $\mu\left(r^{n}\right)=\rho^{n}(n=1,2, \cdots)$, for $r^{n}<m<r^{n-1}$, $\rho^{n}<\mu(m)<\rho^{n-1}(n=1,2, \cdots)$.

A third principle is that, if $\mu$ and $\rho$ are fixed, then g.l.b. ${ }_{\cdot \in \mathfrak{B}_{\mu}} M(\phi, r)$ (which will be denoted by $M(r)$ ) is a continuous, strictly increasing function of $r$ for $0<r<\rho$. Hence the unique extremal functions $\Phi(z ; r)$ of $\mathfrak{B}_{\mu}$ for which

$$
M[\Phi(z ; r), r]=M(r)
$$

are such that, if $r_{0}$ is a given number of the open interval $(0, \rho)$, then $\lim _{r \rightarrow r_{0}} \Phi(z ; r)$ $=\Phi\left(z ; r_{0}\right)$ continuously in the sense of Carathéodory for $|z|<1$.

It is apparent that, if $0<r_{1}<r_{2}<\rho$, then $M\left(r_{1}\right)<M\left(r_{2}\right)$, since

$$
M\left[\Phi\left(z ; r_{1}\right), r_{1}\right] \leqq M\left[\Phi\left(z ; r_{2}\right), r_{1}\right]<M\left[\Phi\left(z ; r_{2}\right), r_{2}\right] .
$$

To establish the continuity of $M(r)$ for $0<r<\rho$, let $r_{0}$ be a given number of this interval. Certainly, if $r<r_{0}, \lim _{r \rightarrow r_{0}} M(r)=M\left(r_{0}\right)$. Otherwise let $\left\{r_{k}\right\}$ be a strictly increasing sequence with limit $r_{0}$ such that $\left\{\Phi\left(z ; r_{k}\right)\right\}$ converges continuously in the sense of Carathéodory for $|z|<1$ as $k \rightarrow \infty$ and let $\Phi^{*}(z)$ denote the limit function of the sequence. If for $r<r_{0}, \lim _{r \rightarrow r_{0}} M(r)<M\left(r_{0}\right)$, 
then $M\left[\Phi^{*}(z), r_{0}\right]<M\left(r_{0}\right)$ and yet $\Phi^{*}(z) \in \mathfrak{B}_{\mu}$. On the other hand, if $r>r_{0}$, $\lim _{r \rightarrow r_{0}} M(r)=M\left(r_{0}\right)$. To prove this, note that for $r_{0}<r<\rho$

$$
M\left[\Phi\left(z ; r_{0}\right), r_{0}\right]<M[\Phi(z ; r), r] \leqq M\left[\Phi\left(z ; r_{0}\right), r\right],
$$

and that given $\epsilon>0$, there exists a positive number $\delta(\epsilon)$ such that, if $0<r-r_{0}$ $<\delta(\epsilon)$,

$$
M\left[\Phi\left(z ; r_{0}\right), r\right]<M\left[\Phi\left(z ; r_{0}\right), r_{0}\right]+\epsilon .
$$

The position of the zeros of $\Phi(z ; r)$. It is apparent that the zeros of $\Phi(z ; r)$ cannot penetrate within some fixed neighborhood of $\rho$ which is independent of $r$. For a given $r$, the following considerations permit one to obtain further information about the position of the zeros. It will be seen later that the present argument is applicable to other extremal problems.

Let $\alpha$ denote a zero of $\Phi(z ; r)$ and suppose that there exists a nonEuclidean straight line $\left.{ }^{4}\right) C$ passing through $\rho$ which separates $\alpha$ from $|z|=r$. It will be shown that this assumption is untenable. If $z_{1}$ and $z_{2}$ are two points of $|z|<1, D\left(z_{1}, z_{2}\right)$ will be used to denote the non-Euclidean distance between them. Let $\beta$ denote the unique point of $|z|<1$ specified by the requirements: (1) $C$ separates $\alpha$ from $\beta$; (2) the non-Euclidean straight line passing through $\alpha$ and $\beta$ is orthogonal to $C$; (3) $D(\alpha, \rho)=D(\beta, \rho)$. Clearly, $C$ is the locus of points of $|z|<1$ with the property that they are equidistant from $\alpha$ and $\beta$ in the non-Euclidean sense. It follows that for $|z|=r, D(\alpha, z)>D(\beta, z)$; and hence that for $|z|=r$

$$
\left|\frac{z-\alpha}{1-\bar{\alpha} z}\right|>\left|\frac{z-\beta}{1-\bar{\beta} z}\right| \text {. }
$$

The alleged extremal function $\Phi(z ; r)$ can be written in the form

$$
\Phi(z ; r)=\frac{z-\alpha}{1-\bar{\alpha} z} g(z),
$$

where $g(z)$ is a $(1, k-1)$ conformal transformation of the interior of the unit circle onto itself. But consider

$$
\Phi^{*}(z) \equiv\left\{\frac{\rho-\alpha}{1-\bar{\alpha} \rho} \cdot \frac{z-\beta}{1-\bar{\beta} z} \cdot g(z)\right\} /\left(\frac{\rho-\beta}{1-\bar{\beta} \rho}\right)
$$

which is a competing function and yet $M\left[\Phi^{*}(z), r\right]<M[\Phi(z ; r), r]$; this is impossible if $\Phi(z ; r)$ is extremal.

Further, there can be no zero of $\Phi(z ; r)$ on the real axis between $r$ and $\rho$. This follows from the readily verified inequality $D\left(r e^{i \theta}, \alpha_{1}\right)<D\left(r e^{i \theta}, \alpha_{2}\right)$, for $\theta$ real and $\alpha_{1}$ and $\alpha_{2}$ real and satisfying $r<\alpha_{1}<\alpha_{2}$. Hence we have the following theorem.

(4) For the concepts of non-Euclidean geometry employed here, see chap. 2 of [3]. 
THEOREM 3.1. No zero of $\Phi(z ; r)$ is separated from $|z|=r$ by a non-Euclidean straight line passing through $\rho$. Further, no zero of $\Phi(z ; r)$ lies on the real axis between $r$ and $\rho$.

4. Results preliminary to studying the degree of an extremal function. The major object of this section and the succeeding one is to study the relation between the degree of an extremal function and the data defining it. The present section contains preliminary results in this direction.

A simple result of this type is the following.

THEOREM 4.1. If $r^{n}<m<r^{n-1}(n=1,2, \cdots)$, and if $f_{0}(z ; m)$ is the corresponding extremal function of $\mathfrak{A}$, then the degree of $f_{0}(z ; m)$ is at least $n$.

Let $k$ denote the degree of $f_{0}(z ; m)$ and assume that $k<n$. It may be assumed that $f_{0}(z ; m)$ does not vanish at the origin; otherwise it would suffice to study $f_{0} / z^{l}$, if $f_{0}$ had an $l$-fold zero at the origin. Actually, it will turn out that under the hypotheses of this theorem, $f_{0}$ does not vanish at the origin. It follows from the theory of $(1, k)$ conformal transformations mapping $|z|<1$ onto itself, from the fact that $f_{0}(z ; m)$ is real for $z$ real, and from Theorem 3.1 that $f_{0}(z ; m)$ can be represented as follows:

$$
f_{0}(z ; m) \equiv \prod_{\lambda=1}^{k} \frac{z-\alpha_{\lambda}}{1-\bar{\alpha}_{\lambda} z} \quad\left[\left|\alpha_{\lambda}\right|<1, \lambda=1,2, \cdots, k\right] .
$$

Hence

$$
f_{0}(1 / \bar{z} ; m) \equiv 1 / \overline{f_{0}(z ; m)} .
$$

Define the rational function $F(z)$ by

$$
F(z) \equiv-z^{k}+f_{0}(z ; m) .
$$

Since $m<r^{n-1} \leqq r^{k}$, it follows from Rouché's theorem that $F(z)$ would have $k$ zeros interior to $|z|=r$. Since $-z^{k}$ shares the property expressed by (4.2) with $f_{0}(z ; m), F(z)$ would have $k$ zeros exterior to $|z|=1 / r$. Observe that $F(1)=0$, and yet by assumption $F(z)$ is of degree $2 k$. The contradiction is manifest.

The degree of $f_{0}(z ; m)\left(r^{n}<m<r^{n-1} ; n=1,2, \ldots\right)$ is at most equal to the number of times, $l,\left|f_{0}(z ; m)\right|$ attains the value $M\left(f_{0}, r\right)$ on $|z|=r$. This follows from the Pick-Nevanlinna theory. But this remark can be strengthened by deleting "at most" and this is the substance of the following theorem.

THEOREM 4.2. With the hypothesis of Theorem 4.1, the degree of $f_{0}(z ; m)$ is equal to the number of times that $\left|f_{0}(z ; m)\right|$ attains the value $M\left(f_{0}, r\right)$ on $|z|=r$.

Let $k$ be the degree of $f_{0}(z ; m)$. From $(4.1)$ it follows that $f_{0}(z ; m)$ can be expressed in the form 


$$
f_{0}(z ; m) \equiv P_{k}(z) / Q_{k}(z),
$$

where $P_{k}(z)$ and $Q_{k}(z)$ are polynomials of degree $k$ in $z, P_{k}(z)$ being the numerator and $Q_{k}(z)$ being the denominator of (4.1). The trigonometric polynomial

$$
\psi_{k}(\theta) \equiv m^{2}\left|Q_{k}\left(r e^{i \theta}\right)\right|^{2}-\left|P_{k}\left(r e^{i \theta}\right)\right|^{2}
$$

is of order $k$, non-negative, and vanishes precisely $l$ times in any $\theta$-interval of the form $\theta_{0} \leqq \theta<\theta_{0}+2 \pi$. Hence by a well known result of the theory of trigonometric polynomials $[13$, p. 39] it follows that $l \leqq k$. The proof is complete.

COROLlary. Under the same hypothesis, $z=0$ cannot be a zero of $f_{0}(z ; m)$.

5. The dependence of the degree of the extremal function upon the data. On the basis of the evidence gathered from the results of the previous sections it is natural to conjecture that the degree of the extremal function $\Phi(z ; r)$ of $\mathfrak{B}_{\mu}$ (it is more convenient to study the $\mathfrak{B}$-problem in this connection) is $n$, if $\rho^{n}<\mu<p^{n-1}(n=2,3, \cdots)$. This conjecture will be established in the present section.

The following lemma is fundamental for the argument( $\left.{ }^{5}\right)$.

LEMma 5.1. Let $\mathfrak{B}_{\mu}^{*}$ consist of those $\phi(z) \in \mathfrak{B}_{\mu}$ which are real for $z$ real. If $\mu$ is sufficiently near one and if $r$ is sufficientiy small, then

$$
\underset{\phi \in \mathbb{P}_{\mu}^{:}}{\text {g.l.b. }}\left|\phi\left(r e^{i \theta}\right)\right|
$$

is attained by the unique function $\phi_{\mu} \in \mathfrak{B}_{\mu}{ }^{*}$ defined by

$$
\frac{\phi_{\mu}-\mu}{1-\mu \phi_{\mu}} \equiv \frac{z-\rho}{1-\rho z}
$$

Clearly, $\phi \in \mathfrak{B}_{\mu}{ }^{*}$ can be written in the form

$$
\phi(z) \equiv \frac{\mu+((z-\rho) /(1-\rho z)) \psi(z)}{1+\mu((z-\rho) /(1-\rho z)) \psi(z)},
$$

where $\psi(z)$ is analytic and of modulus not greater than one for $|z|<1$ and is real for $z$ real. It has already been remarked that for $\theta=0$ and $r<\rho<\mu$ the extremal function of this lemma is defined by (5.1). Note that, since $\psi(z)$ is real for $z$ real, Pick's lemma implies that if $|\psi|<1$ for $|z|<1$, then

$$
\frac{|I \psi|}{1-|\psi|^{2}} \leqq \frac{|I z|}{1-|z|^{2}} .
$$

Let $\phi_{\mu}$ be given by $\psi \equiv \psi_{0}$ in (5.2). It suffices to show that $\psi_{0} \equiv 1$ provided $r$ is sufficiently small and $\mu$ is sufficiently near one. If $\psi_{0}\left(r e^{i \theta}\right)$ is denoted by $u+i v$, it is obvious that, if $\left|\psi_{0}\right| \not \equiv 1$ for $|z|<1$,

(5) Related questions have been considered by Rogosinski [9]. 


$$
\frac{|v|}{1-\left(u^{2}+v^{2}\right)} \leqq \frac{r|\sin \theta|}{1-r^{2}} \text {. }
$$

Clearly, the extreme value of $\phi_{\mu}\left(r e^{i \theta}\right)$ can be attained only when $\left|\psi_{0}\right| \equiv 1$ or, if this is not the case, when equality prevails in (5.4). The locus defined by

$$
\frac{|v|}{1-\left(u^{2}+v^{2}\right)}=\frac{r|\sin \theta|}{1-r^{2}} \quad(\theta \neq 00(\bmod \pi))
$$

in $|u+i v| \leqq 1$ consists of two circular arcs passing through -1 and +1 (appropriate conventions being understood at the end points), the one being the reflection of the other with respect to the real axis. Hence, the image of this locus with respect to

$$
u+i v \mid \frac{\mu+A(r, \theta)(u+i v)}{1+\mu A(r, \theta)(u+i v)}
$$

where

$$
A(r, \theta) \equiv \frac{r e^{i \theta}-\rho}{1-\rho r e^{i \theta}}
$$

consists of two circular arcs lying in the interior of the unit circle and having end points in common. Observe that for $r$ sufficiently small

$$
\left|\frac{\mu+A(r, \theta)}{1+\mu A(r, \theta)}\right|<\left|\frac{\mu-A(r, \theta)}{1-\mu A(r, \theta)}\right|
$$

uniformly with respect to $\theta$. Hence it is sufficient to show that on each arc defined by (5.5) the value

$$
\left|\frac{\mu+A(r, \theta)(u+i v)}{1+\mu A(r, \theta)(u+i v)}\right|^{2}
$$

has a relative minimum at $u+i v=+1$. To study (5.6) for values of $u+i v$ satisfying (5.5) and near one, set $u=1-\tau$. It follows that $|v|$ is asymptotically given by

$$
|v| \sim \frac{2 r|\sin \theta|}{1-r^{2}} \tau
$$

It now suffices to show that the derivative of

(5.8) $\sigma(\tau) \equiv\left|\frac{\mu+A(r, \theta)(1-\alpha \tau)}{1+\mu A(r, \theta)(1-\alpha \tau)}\right|^{2}, \quad$ where $\quad \alpha=1 \pm \frac{2 r|\sin \theta|}{1-r^{2}} i$, 
is positive for $\tau=0$. Observe that

$$
\begin{aligned}
\sigma(\tau) & =\left|\frac{\mu+A(r, \theta)}{1+\mu A(r, \theta)}\right|^{2} \cdot\left|\frac{1-\alpha A(r, \theta) \tau /(\mu+A(r, \theta))}{1-\mu \alpha A(r, \theta) \tau /(1+\mu A(r, \theta))}\right|^{2} \\
& =\left|\frac{\mu+A(r, \theta)}{1+\mu A(r, \theta)}\right|^{2} \cdot\left|1+\alpha A(r, \theta) \tau\left(\frac{\mu}{1+\mu A(r, \theta)}-\frac{1}{\mu+A(r, \theta)}\right)+\cdots\right|^{2} .
\end{aligned}
$$

Hence

$$
\sigma^{\prime}(0)=2\left|\frac{\mu+A(r, \theta)}{1+\mu A(r, \theta)}\right|^{2} R\left\{\frac{\alpha A(r, \theta)\left(\mu^{2}-1\right)}{(1+\mu A(r, \theta))(\mu+A(r, \theta))}\right\} ;
$$

and since

$$
\operatorname{sgn} \sigma^{\prime}(0)=-\operatorname{sgn} R\left\{\frac{\alpha A(r, \theta)}{(1+\mu A(r, \theta))(\mu+A(r, \theta))}\right\},
$$

it suffices to show that

$$
R\left\{\frac{\alpha A(r, \theta)}{(1+\mu A(r, \theta))(\mu+A(r, \theta))}\right\}
$$

is negative for $r$ sufficiently small and $\mu$ sufficiently near one. Note that (5.10) is continuous in $r$ and $\mu$ at $r=0$ and $\mu=1$ and that for $r=0, \mu=1,(5.10)$ reduces to $-\rho /(1-\rho)^{2}$.

The proof of the lemma is complete.

Actually, precise relations between $\mu, \rho$ and $r$ may be found which guarantee that $\sigma^{\prime}(0)<0$, but these will not be considered here.

The proof of the conjecture concerning the degree of $\Phi(z ; r)$ is now easy provided that $r$ is such that the conclusion of Lemma 5.1 is true for $\mu$ sufficiently near one. Let $\mu$ satisfy $\rho^{n}<\mu<\rho^{n-1}(n=2,3, \cdots)$. If $\Phi(z ; r, \mu)$ is the corresponding extremal function, it follows from the results of $\S 3$ that for $\mu$ sufficiently near $\rho^{n-1}, \Phi(z ; r, \mu)$ has in the neighborhood of $z=0$ a set of zeros $\alpha_{1}(\mu), \alpha_{2}(\mu), \cdots, \alpha_{N}(\mu)$ of respective multiplicities $k_{1}(\mu), k_{2}(\mu), \cdots, k_{N}(\mu)$ with $k_{1}+k_{2}+\cdots+k_{N}=n-1$. Let $\theta(z ; \mu)$ be defined by

$$
\theta(z ; \mu) \equiv \prod_{\nu=1}^{N}\left[\frac{z-\alpha_{\nu}(\mu)}{1-\overline{\alpha_{\nu}(\mu)} z}\right]^{k_{\nu}} .
$$

Since $\Phi(z ; r, \mu)$ is real for $z$ real, it follows that $\theta(z ; \mu)$ is also. Hence $\theta(\rho ; \mu)$ is positive. Further, it follows from Theorem 4.1 and $\S 3$ that $\theta(\rho ; \mu)>\mu$. Let $\mu^{*}$ denote $\mu / \theta(\rho ; \mu)$. For $\mu$ sufficiently near $\rho^{n-1}, \mu^{*}$ is arbitrarily close to one. Apply Lemma 5.1 with $\mu^{*}$ replacing $\mu$ of that lemma. Clearly,

$$
\left|\frac{\Phi\left(r e^{i \theta} ; r, \mu\right)}{\theta\left(r e^{i \theta}, \mu\right)}\right| \geqq\left|\phi_{\mu^{*}}\left(r e^{i \theta}\right)\right|
$$


and hence, since $\Phi(z ; r, \mu)$ is extremal, it must necessarily be equal to $\theta(z ; \mu) \phi_{\mu^{*}}(z)$. Thus for values of $\mu<\rho^{n-1}$ but sufficiently near $\rho^{n-1}$, the degree of $\Phi(z ; r, \mu)$ is precisely $n$. Let $\mu_{1}$ be the least positive number satisfying: (1) $\mu_{1} \geqq \rho^{n} ;(2)$ for $\mu_{1}<\mu<\rho^{n-1}$ the degree of $\Phi(z ; r, \mu)$ is precisely $n$. Since $\Phi(z ; r, \mu)$ depends continuously on $\mu$, it follows that $\Phi\left(z ; r, \mu_{1}\right)$ is also of degree $n$. It suffices to show that $\mu_{1}=\rho^{n}$. Suppose that this is not the case. Since for values of $\mu$ less than $\mu_{1}$, but sufficiently near $\mu_{1}$, in arbitrarily assigned neighborhoods of the zeros of $\Phi\left(z ; r, \mu_{1}\right), \Phi(z ; r, \mu)$ has zeros of the same total multiplicity as $\Phi\left(z ; r, \mu_{1}\right)$, a repetition of the argument just applied to $\mu$ near $\rho^{n-1}$ shows that for some positive $\epsilon, \Phi(z ; r, \mu)$ would be of degree $n+1$ for $\mu_{1}-\epsilon \leqq \mu<\mu_{1}$. That this situation is impossible will follow from Theorem 4.2.

Recall (4.4) and (4.5) and observe that for $\mu_{1}-\epsilon \leqq \mu<\mu_{1}$,

$$
\Phi(z ; r, \mu) \equiv P_{\mu, n+1}(z) / Q_{\mu, n+1}(z),
$$

where

$$
P_{\mu, n+1}(z) \equiv \prod_{k=1}^{n+1}\left(z-\alpha_{k}(\mu)\right)
$$

and

$$
Q_{\mu, n+1}(z) \equiv \prod_{k=1}^{n+1}\left(1-\overline{\alpha_{k}(\mu)} z\right),
$$

the $\alpha_{k}(\mu)$ being the zeros of $\Phi(z ; r ; \mu)$. Further, let $\Phi\left(z ; r, \mu_{1}\right)$ be written in the form $P_{\mu_{1}, n}(z) / Q_{\mu_{1}, n}(z)$, with $P_{\mu_{1}, n}$ and $Q_{\mu_{1}, n}$ defined in the manner of $P_{\mu, n+1}$, $Q_{\mu, n+1}$. The coefficients of $P_{\mu, n+1}$ and $Q_{\mu, n+1}$ are uniformly bounded independent of $\mu$ in $\left(\mu_{1}-\epsilon \leqq \mu<\mu_{1}\right)$. Since under the assumption made one of the zeros of $\Phi(z ; r, \mu)$ would tend to -1 as $\mu \rightarrow \mu_{1}\left(\mu<\mu_{1}\right)$, it follows that

$$
\begin{aligned}
\lim _{\mu \rightarrow \mu_{1}}\left\{\left|P_{\mu, n+1}\left(r e^{i \theta}\right)\right|^{2}-\left|m(\mu) Q_{\mu, n+1}\left(r e^{i \theta}\right)\right|^{2}\right\} \\
=\left|1+r e^{i \theta}\right|^{2}\left\{\left|P_{\mu_{1}, n}\left(r e^{i \theta}\right)\right|^{2}-\left|m\left(\mu_{1}\right) Q_{\mu_{1}, n}\left(r e^{i \theta}\right)\right|^{2}\right\} .
\end{aligned}
$$

This is impossible since $\left|1+r e^{i \theta}\right|^{2}$ does not vanish for $\theta$ real and since therefore by Theorem 4.2 the right-hand member of (5.14) accounts for precisely $n$ double zeros in a $\theta$-interval of the form $\left(\theta_{0} \leqq \theta<\theta_{0}+2 \pi\right)$, whereas the expression inside the brackets on the left-hand side of (5.14) always accounts for precisely $n+1$ double zeros in a $\theta$-interval of the same type, when $\mu_{1}-\epsilon \leqq \mu<\mu_{1}$. Hence $\mu_{1}=\rho^{n}$, and the proof is complete.

THEOREM 5.1. If $\rho^{n}<\mu<\rho^{n-1}$ and $r$ is sufficiently small, then the degree of $\Phi(z ; r, \mu)$ is precisely $n$.

The restriction on the size of $r$ can be dropped by the following argument. 
Consider the function $\left(^{6}\right) \Theta(z ; r, \mu)$ defined by

$$
\Theta(z ; r, \mu) \equiv \Phi(z ; r, \mu) \Phi\left(r^{2} / z ; r, \mu\right) .
$$

Clearly, $\Theta(z ; r, \mu)$ is analytic and of modulus less than unity for $r^{2} \leqq|z| \leqq 1$ and is real and positive for $|z|=r$ since $\Phi(z ; r, \mu)$ is real for $z$ real. At the points of $|z|=r$ where $|\Phi(z ; r, \mu)|=m(r, \mu)\left(m(r, \mu)=\max _{|z|=r}|\Phi(z ; r, \mu)|\right)$ the function $\Theta^{*}(z ; r, \mu) \equiv \Theta(z ; r, \mu)-[m(r, \mu)]^{2}$ has double zeros since these points yield relative maxima of $\Theta(z ; r, \mu)$ on the circle $|z|=r$. Since the degree of $\Theta^{*}(z ; r, \mu)$ is twice the degree of $\Phi(z ; r, \mu)$, it follows that $\Theta^{*}(z ; r, \mu)$ can have no other zeros.

Now suppose that $r_{0}$ is the least upper bound of those $r$ for which the degree of $\Phi(z ; r, \mu)$ is $n$ ( $\mu$ being fixed and satisfying the hypothesis of Theorem 5.1). If $r_{0}=\rho$, then the desired extension of Theorem 5.1 would be established. Suppose therefore that $r_{0}<\rho$. The continuity of $\Phi(z ; r, \mu)$ with respect to $r$ $(0<r<\rho)$ and Theorem 4.1 imply that the degree of $\Phi\left(z ; r_{0}, \mu\right)$ is precisely $n$. Consider now values of $r$ which are slightly larger than $r_{0}$. From the continuity of $m(r, \mu)$ and $\Phi(z ; r, \mu)$ with respect to $r$, the properties of $\Theta^{*}(z ; r, \mu)$ already enumerated, and Hurwitz's theorem, it follows that for the $r$ under consideration the degree of $\Theta^{*}(z ; r, \mu)$ is $2 n$ and hence the degree of $\Phi(z ; r, \mu)$ is $n$. It follows that $r_{0}=\rho$. Hence we have the following theorem.

THEOREM 5.2. If $\rho^{n} \leqq \mu<\rho^{n-1}$, then the degree of $\Phi(z ; r, \mu)$ is precisely $n$.

6. Examination of the case where $\rho^{2}<\mu<\rho$. In this section the location of the zeros of $\Phi(z ; r, \mu)$ is studied in the special case $\rho^{2}<\mu<\rho$. It will also be shown how $\Phi(z ; r, \mu)$ may be calculated in terms of the data.

First, note as in $\$ 5$ that for $\mu<\rho$, but sufficiently near $\rho, \Phi(z ; r, \mu)$ has a simple real zero in the neighborhood of $z=0$. This zero must be positive, for otherwise the argument of $\S 5$ would prove that $\Phi(z ; r, \mu)$ would be of the second degree and would have two negative zeros. But then $\left|\Phi\left(r e^{i \theta} ; r, \mu\right)\right|$ $(\theta$ real $)$ would attain its maximum only at $\theta \equiv 0(\bmod 2 \pi)$ and this contradicts Theorem 4.2. The second zero of $\Phi$ is negative and near -1 . By employing the typical continuity argument of the previous sections and by recalling the Corollary of Theorem 4.2 , it may be shown that for all $\mu$ between $\rho^{2}$ and $\rho, \Phi(z ; \mu, r)$ has one simple positive zero and one simple negative zero.

Further, $\left|\Phi\left(r e^{i \theta} ; r, \mu\right)\right|(\theta$ real $)$ cannot attain its maximum at either $\theta \equiv 0$ $(\bmod 2 \pi) \operatorname{or} \theta \equiv \pi(\bmod 2 \pi)$. For it follows from Theorem 4.2 that if the maximum is attained for one of these sets of $\theta$ it must be attained for the other. On the other hand, if this situation were to occur for some $\mu$, say $\mu_{0}$, between $\rho^{2}$ and $\rho$, then it would be true for all $\mu$ subject to $\rho^{2}<\mu<\rho$. This fact may be established by studying the trigonometric polynomial (4.5) for the present

(6) I am indebted to Professor R. M. Robinson for suggesting the use of the function $\Theta(z ; r, \mu)$ in this connection. 
situation and observing that if $\left|\Phi\left(r e^{i \theta} ; r, \mu\right)\right|$ assumed its maximum at $\theta \equiv 0$ $(\bmod 2 \pi)$ and $\theta \equiv \pi(\bmod 2 \pi)$ for one value of $\mu$ between $\rho^{2}$ and $\rho$, and for another such $\mu$ at $\theta \neq \equiv 0(\bmod \pi)$, then there would be values of $\mu$ between $\rho^{2}$ and $\rho$ for which the derivative of (4.5) would have at least five zeros in a $\theta$-interval of the form $\theta_{0} \leqq \theta<\theta_{0}+2 \pi$. It is well known that this is impossible. Let $\alpha(\mu)$ be the positive zero and $\beta(\mu)$ be the negative zero of $\Phi(z ; r, \mu)$. Note that $\alpha(\mu)$ and $\beta(\mu)$ depend continuously on $\mu$ and that $\beta(\mu)$ is near -1 for $\mu$ near $\rho$ and is near 0 for $\mu$ near $\rho^{2}$. Hence there is a value of $\mu$ such that $\beta(\mu)=-r$, and in this case it is impossible for $\left|\Phi\left(r e^{i \theta} ; r, \mu\right)\right|$ to attain its maximum at $\theta \equiv \pi(\bmod 2 \pi)$.

Computation of $\Phi(z ; r, \mu)$. Set

Clearly,

$$
\Phi(z ; r, \mu) \equiv \frac{z-\alpha(\mu)}{1-\alpha(\mu) z} \cdot \frac{z-\beta(\mu)}{1-\beta(\mu) z} .
$$

$$
\begin{aligned}
\left|m(\mu)\left(1-\alpha r e^{i \theta}\right)\left(1-\beta r e^{i \theta}\right)\right|^{2}-\left|\left(r e^{i \theta}-\alpha\right)\left(r e^{i \theta}-\beta\right)\right|^{2} \\
\equiv \\
=[m(\mu)]^{2}\left\{\left(1+\alpha^{2} r^{2}-2 \alpha r \cos \theta\right)\left(1+\beta^{2} r^{2}-2 \beta r \cos \theta\right)\right\} \\
-\left\{\left(r^{2}+\alpha^{2}-2 \alpha r \cos \theta\right)\left(r^{2}+\beta^{2}-2 \beta r \cos \theta\right)\right\} .
\end{aligned}
$$

If in (6.2) $\cos \theta$ is set equal to $x$, a polynomial

$$
A x^{2}+2 B x+C
$$

is obtained, which is non-negative for $-1<x<1$ and vanishes at a single value on this interval, say $x_{0}$. Since $A>0, A, B, C$ must satisfy

$$
B^{2}-A C=0 \text {, }
$$

where $A, B, C$ are given by

$$
\begin{aligned}
A & =4 \alpha \beta\left([m(\mu)]^{2}-1\right) r^{2}, \\
(6.5) \quad B & =(\alpha+\beta) r\left\{\left(1-\alpha \beta[m(\mu)]^{2}\right) r^{2}+\left(\alpha \beta-[m(\mu)]^{2}\right)\right\}, \\
C & =\left(\alpha^{2} \beta^{2}[m(\mu)]^{2}-1\right) r^{4}+\left([m(\mu)]^{2}-1\right)\left(\alpha^{2}+\beta^{2}\right) r^{2}+\left([m(\mu)]^{2}-\alpha^{2} \beta^{2}\right) .
\end{aligned}
$$

Now $\beta(\mu)$ can be expressed in terms of $\alpha(\mu)$ by

$$
\frac{\rho-\alpha(\mu)}{1-\alpha(\mu) \rho} \cdot \frac{\rho-\beta(\mu)}{1-\beta(\mu) \rho}=\mu,
$$

and from (6.4) $[m(\mu)]^{2}$ can be expressed in terms of $\alpha, r, \rho, \mu$ and one need find only that value of $\alpha$ between zero and $\rho$ making $m^{2}$ least.

7. A related extremal problem. Referring to the class $\mathfrak{B}_{\mu}$ of functions $\phi(z)$ defined in $\$ 3$, preserving the same notation and letting $E_{r}$ denote the interval $(-r \leqq x \leqq r<1)(x$ real $)$, consider the following extremal problem:

To determine g.l.b. ${ }_{\phi \in \mathfrak{B}_{\mu}}\left\{\max _{x \in E_{r}}|\phi(x)|\right\}$ and the associated extremal functions. 
Clearly, the dual to this problem is to determine l.u.b. $\downarrow \in \mathfrak{G}_{m} \psi(\rho)$ and the associated extremal functions of $\mathfrak{S}_{m}$, where $\mathfrak{S}_{m}$ is defined to be the class of functions $\psi(z)$ which are analytic and of modulus less than unity for $|z|<1$, and satisfy the further requirements that for a given positive number $m$, less than one, $\max _{x \in E_{r}}|\psi(x)| \leqq m$, and that $\psi(\rho)$ is real. The object of this section is to study the descriptive properties of these extremal functions.

The methods of $\$ \$ 2-4$ apply without essential modification and the following first result may be stated without proof.

THEOREM 7.1. The extremal functions for the problems just proposed are unique and define $(1, k)$ ( $k$ depending upon the parameters of the respective problem considered) conformal maps of the interior of the unit circle onto itself. Further, they are real for $z$ real. The totality of extremal functions $\phi_{0}(z ; \mu)\left(\in \mathfrak{B}_{\mu}\right)$, where all values of $\mu$ between zero and one are considered, is the same as the totality of extremal functions $\psi_{0}(z ; m)\left(\in \mathfrak{\mho}_{m}\right)$, where all values of $m$ between zero and one are considered.

The extremal function $\phi_{0}(z ; \mu)$ depends continuously in the sense of Carathéodory on $\mu$ for $|z|<1$ (cf. §3) and similarly $\psi_{0}(z ; m)$ depends continuously in the same sense on $m$ for $|z|<1$.

Because of this theorem for the remainder of the present section attention will be confined to the extremal problem for $\mathfrak{B}_{\mu}$. A first result in the study of the descriptive properties of the extremal function $\phi_{0}(z ; \mu)$ is the following:

TheOREM 7.2. All the zeros of $\phi_{0}(z ; \mu)$ are real and less than $r$.

This follows on applying the argument of Theorem 3.1.

Another simple result is that $\phi_{0}(z ; \mu)$ admits no multiple zeros. To establish this, consider the following simple lemma.

LEMMA 7.1. Let $\alpha_{1}, \alpha_{2}, \beta_{1}, \beta_{2}$ be real and satisfy the two requirements:

$$
\begin{aligned}
&-1<\alpha_{1}<\alpha_{2} \leqq \beta_{2}<\beta_{1}<\rho ; \\
& \frac{\rho-\alpha_{1}}{1-\alpha_{1} \rho} \cdot \frac{\rho-\beta_{1}}{1-\beta_{1} \rho}=\frac{\rho-\alpha_{2}}{1-\alpha_{2} \rho} \cdot \frac{\rho-\beta_{2}}{1-\beta_{2} \rho} .
\end{aligned}
$$

Then for $-1<x<\rho$,

$$
\frac{x-\alpha_{1}}{1-\alpha_{1} x} \cdot \frac{x-\beta_{1}}{1-\beta_{1} x}<\frac{x-\alpha_{2}}{1-\alpha_{2} x} \cdot \frac{x-\beta_{2}}{1-\beta_{2} x} .
$$

To establish this lemma, let $r_{k}(x)$ denote

$$
\frac{x-\alpha_{k}}{1-\alpha_{k} x} \cdot \frac{x-\beta_{k}}{1-\beta_{k} x}
$$$$
(k=1,2) \text {. }
$$

Observe that $R(x) \equiv r_{2}(x)-r_{1}(x)$ is rational and of the fourth degree. Since 
$r_{k}(1 / x) \equiv 1 / r_{k}(x)(k=1,2)$ and since $R(-1)=R(1)=R(\rho)=R(1 / \rho)=0, R(x)$ vanishes for no other $x$. Hence the lemma follows.

If $\phi_{0}(z ; \mu)$ admitted a multiple zero, by Lemma 7.1 it could be replaced by a function $\Phi(z) \in \mathfrak{B}_{\mu}$ such that

$$
\max _{x \in E_{r}}|\Phi(x)|<\max _{x \in E_{r}}\left|\phi_{0}(x ; \mu)\right|
$$

and $\phi_{0}(z ; \mu)$ would not be extremal. Hence we have the following theorem.

THEOREM 7.3. The extremal function $\phi_{0}(z ; \mu)$ admits no multiple zeros.

These results and the following one will be quite useful in studying the degree of $\phi_{0}(z ; \mu)$ and the location of its zeros.

ThEOREM 7.4. Let $m(\mu)$ denote g.l.b. $\phi_{\phi \in \mathscr{P}_{\mu}}\left\{\max _{x \in E_{r}}|\phi(x)|\right\}$. Then $\phi_{0}(r ; \mu)$ $=m(\mu)$.

Let $\epsilon$ be positive and less than one and let $T_{p, \epsilon}(z)$ be defined by

$$
T_{\rho, \mathrm{e}}(z): \frac{1-T_{\rho, \mathrm{e}}}{1+T_{\rho, \mathrm{e}}} \equiv\left(\frac{1-\rho}{1+\rho}\right)^{e} \cdot\left(\frac{1-z}{1+z}\right)^{1-\epsilon},
$$

where the determinations of $Z^{\bullet}$ and $Z^{1-\bullet}$ which are positive for $Z$ positive are used. The function $T_{p, e}(z)$ is defined and analytic for $|z|<1$; further $\left|T_{\rho, \mathrm{e}}\right|<1$ for $|z|<1$ and $T_{\rho, \epsilon}(\rho)=\rho$. It follows from Pick's lemma that

$$
T_{\rho, \epsilon}(x)>x \text { for }-1<x<\rho .
$$

To establish Theorem 7.4 note that by Theorem 7.2 and the fact that $\phi_{0}(\rho ; \mu)=\mu, \phi_{0}(r ; \mu)>0$. If $\phi_{0}(r ; \mu)<m(\mu)$, for $\epsilon$ sufficiently small $\phi_{0}\left[T_{\rho, e}(z) ; \mu\right]$, which belongs to $\mathfrak{B}_{\mu}$, would be such that

$$
\max _{x \in E_{r}}\left|\phi_{0}\left[T_{\rho, e}(x) ; \mu\right]\right| \leqq \max _{x \in E_{r}}\left|\phi_{0}(x ; \mu)\right| \text {. }
$$

By Theorem 7.1, $\phi_{0}\left[T_{p, e}(z) ; \mu\right] \equiv \phi_{0}(z ; \mu)$, and this is impossible for $\epsilon$ positive.

Let $k$ denote the degree of $\phi_{0}(z ; \mu)$ and let $\alpha_{1}, \alpha_{2}, \cdots, \alpha_{k}$ denote the zeros of $\phi_{0}(z ; \mu)$, where

$$
-1<\alpha_{1}<\alpha_{2}<\cdots<\alpha_{k}<r .
$$

For $x$ real and strictly between $\alpha_{1}$ and $\alpha_{k}, \phi_{0}(z ; \mu)$ admits at least $k-1$ (nonvanishing) relative maxima and minima. There can be no more than $k-1$ relative maxima or minima with $x$ strictly between -1 and 1 . This is established by noting that

$$
\phi_{0}(1 / x ; \mu)=1 / \phi_{0}(x ; \mu)
$$

and that the numerator of $\phi_{0}^{\prime}(x ; \mu)$ is at most of degree $2 k-2$. Hence there are precisely $k-1$ relative maxima and minima for $\phi_{0}(x ; \mu)$ with $-1<x<1$. Let $\xi_{\lambda}$ denote the position of the relative maximum or minimum for which 
$\alpha_{\lambda}<\xi_{\lambda}<\alpha_{\lambda+1}(\lambda=1,2, \cdots, k-1)$. Lemma 7.1 permits the inference that, if $-r \leqq \xi_{\lambda}<r$, then $\left|\phi_{0}\left(\xi_{\lambda} ; \mu\right)\right|=m(\mu)$. Otherwise $\left|\phi_{0}\left(\xi_{\lambda} ; \mu\right)\right|<m(\mu)$. Let $\alpha_{\lambda}$ and $\alpha_{\lambda+1}$ be replaced by $\alpha_{\lambda}^{*}$ and $\alpha_{\lambda+1}^{*}$ subject to the restrictions that (1) $\alpha_{\lambda}^{*}<\alpha_{\lambda}$ $<\alpha_{\lambda+1}<\alpha_{\lambda^{*}+1}^{*}$

$$
\frac{\rho-\alpha_{\lambda}^{*}}{1-\alpha_{\lambda}^{*} \rho} \cdot \frac{\rho-\alpha_{\lambda+1}^{*}}{1-\alpha_{\lambda+1}^{*} \rho}=\frac{\rho-\alpha_{\lambda}}{1-\alpha_{\lambda} \rho} \cdot \frac{\rho-\alpha_{\lambda+1}}{1-\alpha_{\lambda+1} \rho}
$$

(3) $\alpha_{\lambda}^{*}$ is so near $\alpha_{\lambda}$ and $\alpha_{\lambda+1}^{*}$ is so near $\alpha_{\lambda+1}$ that the function obtained by replacing the factors $\left(z-\alpha_{\lambda}\right) /\left(1-\alpha_{\lambda} z\right)$ and $\left(z-\alpha_{\lambda+1}\right) /\left(1-\alpha_{\lambda+1} z\right)$ in $\phi_{0}(z ; \mu)$ by $\left(z-\alpha_{\lambda}^{*}\right) /\left(1-\alpha_{\lambda}^{*} z\right)$ and $\left(z-\alpha_{\lambda}^{*}+1\right) /\left(1-\alpha_{\lambda}^{*} z\right)$ respectively is in absolute value less than $m(\mu)$ at $x=\xi_{\lambda}$. It follows from Lemma 7.1 that $\phi_{0}(z ; \mu)$ could not be extremal.

Let $l$ denote the number of positions on the interval $E_{r}$, where $\left|\phi_{0}(x ; \mu)\right|$ $=m(\mu)$. From the Pick-Nevanlinna interpolation theory, it follows that $k \leqq l$. Let $K$ denote the number of $\alpha_{\lambda}$ in the interval $E_{r}$. Then since $\phi_{0}(r ; \mu)=m(\mu)$ and since $\left|\phi_{0}(-r ; \mu)\right|$ may possibly attain the value $m(\mu), l \leqq 2+(K-1)$ $\leqq 2+(k-1)=k+1$. Hence $K \geqq k-1$. At least $k-1$ of the $\alpha_{\lambda}$ are in $E_{r}$. That $l$ can equal $k+1$ will be seen presently. One immediate consequence of these observations is that at successive points of $E_{r}$ where $\left|\phi_{0}(x ; \mu)\right|=m(\mu)$ the respective signs of $\phi_{0}(x ; \mu)$ are opposite. Hence the following theorem holds.

THEOREM 7.5. If $\phi_{0}(z ; \mu)$ is of degree $k,\left|\phi_{0}(x ; \mu)\right|$ attains $m(\mu)$ at least $k$ times, at most $k+1$ times on $E_{r}$. At successive points where $\left|\phi_{0}(x ; \mu)\right|=m(\mu)$, the respective signs of $\phi_{0}(x ; \mu)$ are opposite. At most one zero of $\phi_{0}(z ; \mu)$ lies to the left of $x=-r$.

By use of this theorem an estimate for the degree of $\phi_{0}(z ; \mu)$ in terms of $\mu$ may be obtained quite simply. Observe that at least one zero of $\phi_{0}(z ; \mu)$ is not positive, since $\left|\phi_{0}(-r ; \mu)\right| \leqq \phi_{0}(r ; \mu)$, and that there are at least $k-1$ zeros of $\phi_{0}(z ; \mu)$ in $E_{r}$. Hence

$$
\rho\left(\frac{\rho-r}{1-\rho r}\right)^{k-1}<\mu<\left(\frac{\rho+r}{1+\rho r}\right)^{k-1} ;
$$

and hence

$$
\log \frac{\rho}{\mu} / \log \left(\frac{1-\rho r}{\rho-r}\right)<k-1<\log \frac{1}{\mu} / \log \left(\frac{1+\rho r}{\rho+r}\right) .
$$

How do $\phi_{0}(z ; \mu)$ and its degree $k(\mu)$ vary as $\mu$ decreases from one to zero? The following lemma will be of service in discussing this question.

LEMMA 7.2. For each $k(k=1,2, \cdots)$ there exists $a(1, k)$ directly conformal mapping $\pi_{k}(z)$ of $|z|<1$ onto itself which satisfies the following conditions: (1) all the zeros of $\pi_{k}(z)$ are simple and lie in the interior of $E_{r} ;(2) \max _{x} \in E_{r}\left|\pi_{k}(x)\right|$ 
is attained at $k+1$ distinct points of $E_{r}$ including $-r$ and $r$ by $\left|\pi_{k}(x)\right| ;(3) \pi_{k}(r)$ is real and positive. The function $\pi_{k}(z)$ is the only $(1, k)$ directly conformal mapping of $|z|<1$ onto itself which satisfies these conditions. Hence $\max _{x} \in E_{r}$ $\left|\pi_{k}(x)\right|$ depends only on $k$ and $r$.

The proof of the existence statement will be omitted inasmuch as it is not essential for the argument that follows $\left({ }^{7}\right)$. Uniqueness may be established by studying the ramification properties of the covering of $|w|<1$ defined by $w=\pi_{k}(z)$. Properties (1) and (2) of $\pi_{k}(z)$ imply that $\pi_{k}(z)$ is locally $(1,1)$ over every point of $|w|<1$ except possibly the points $w_{1}=\max _{x \in E_{r}}\left|\pi_{k}(x)\right|$ and $w_{2}=-\max _{x \in E_{r}}\left|\pi_{k}(x)\right|$. The behavior of the mapping over $w_{1}$ and $w_{2}$ may be readily inferred from (1), (2), (3). At all events if $\pi_{k}^{(1)}(z)$ and $\pi_{k}^{(2)}(z)$ both satisfy (1), (2), (3) and in addition

$$
\max _{x \in E_{r}}\left|\pi_{k}^{(1)}(x)\right|=\max _{x \in E_{r}}\left|\pi_{k}^{(2)}(x)\right|,
$$

then the Riemann domains lying over $|w|<1$ which are defined by $\pi_{k}^{(1)}(z)$ and $\pi_{k}^{(2)}(z)$ are identical and the condition (3) guarantees $\pi_{k}^{(1)}(z) \equiv \pi_{k}^{(2)}(z)$. Suppose now that

$$
\max _{x \in E_{r}}\left|\pi_{k}^{(1)}(x)\right|<\max _{x \in E_{r}}\left|\pi_{k}^{(2)}(x)\right|
$$

and denote the left and right members of this inequality by $m_{1}$ and $m_{2}$ respectively. The Riemann domain which is the image of $|z|<1$ with respect to $w=\pi_{k}^{(1)}(z)$ would be a continuation of the Riemann domain which is the image of $|z|<1$ with respect to $w=\left(m_{1} / m_{2}\right) \pi_{k}^{(2)}(z)$. Hence there exists a function $\theta(z)$, analytic and univalent for $|z| \leqslant 1$, which maps $|z|<1$ into itself and satisfies

$$
\left(m_{1} / m_{2}\right) \pi_{k}^{(2)}(z) \equiv \pi_{k}^{(1)}[\theta(z)], \quad \theta(r)=r .
$$

Observe that the derivative of the left member of (7.8) is different from zero at $z=-r$ by virtue of condition (1). Hence $\theta(-r)$ must equal $-r$ since $\theta(r)=r$ and $\theta(z)$ is univalent. This is clearly impossible unless $\theta \equiv z$ and this can happen only when $m_{1}=m_{2}$. The last statement of the lemma is now evident.

To return to the study of $\phi_{0}(z ; \mu)$ and $k(\mu)$, if $\rho \leqq \mu<1$, the argument used in connection with the Three Circles Theorem problem shows that $\phi_{0}(z ; \mu)$ is defined by

$$
\frac{\mu-\phi_{0}(z ; \mu)}{1-\mu \phi_{0}(z ; \mu)} \equiv \frac{\rho-z}{1-\rho z} .
$$

Thus for $\rho \leqq \mu<1, k(\mu)=1$. But for $\mu<\rho$, this cannot be the case because Pick's lemma would imply $\left|\phi_{0}(-r ; \mu)\right|>\phi_{0}(r ; \mu)$. Next, let $B(x ; \mu)$ denote

$\left({ }^{7}\right)$ A forthcoming paper of the author will treat the properties of the $\pi_{k}(z)$ which are analogues of the Tchebycheff polynomials in the hyperbolic plane. 
g.l.b. $\phi \in \mathfrak{F}_{\mu}|\phi(x ; \mu)|$ where $x \in E_{r}$, and consider values of $\mu$ lying between $(\rho+r) /(1+\rho r)$ and one. For such $\mu, B(x ; \mu)$ is equal to $\phi_{0}(x ; \mu)$ as defined by (7.9).

Recall the last statement of Theorem 7.1 and consider values of $\mu$ less than but very close to $\rho$. Since $\phi_{0}(z ; \rho) \equiv z$ which has a simple zero at $z=0$, it follows from Theorem 7.1 and Hurwitz's theorem that for values of $\mu$ under consideration, $\phi_{0}(z ; \mu)$ has a simple zero in the neighborhood of $z=0$, say $\alpha(\mu)$. But then $\mu^{*}=\mu[(\rho-\alpha(\mu)) /(1-\rho \alpha(\mu))]^{-1}$ would be very close to one and it follows that for $x \in E_{\text {r }}$

$$
\left|\phi_{0}(x ; \mu)\right| \geqq\left|\frac{x-\alpha(\mu)}{1-\alpha(\mu) x}\right| \phi_{0}\left(x ; \mu^{*}\right) .
$$

This implies that actually $\phi_{0}(z ; \mu) \equiv \phi_{0}\left(z ; \mu^{*}\right)(z-\alpha(\mu)) /(1-\alpha(\mu) z)$, and hence for $\mu<\rho$ but close to $\rho, k(\mu)=2$. For such $\mu, \phi_{0}(z ; \mu)$ has a zero near the origin and another near -1 . Let $\mu_{2}$ denote the greatest lower bound of the set of $\mu$ for which not only $k(\mu)=2$, but also there exists no $\mu^{*}$ with $k\left(\mu^{*}\right)>2$ separating $\mu$ from $\rho$. From Theorem 7.1 and the fact that the degree of $\lim _{\mu \rightarrow \mu_{2}} \phi_{0}(z ; \mu)$ is at most two and cannot equal one, it follows that $k\left(\mu_{2}\right)=2$. To continue the examination of $k(\mu)$, consider values of $\mu$ less than but near $\mu_{2}$. A repetition of the argument used for $\mu$ less than but near $\rho$ shows that in the present case $k(\mu)=3$ and that one zero of $\phi_{0}(z ; \mu)$ is near -1 and two are in the interval $E_{r}$. Now let $\mu_{3}$ denote the greatest lower bound of the set $\{\mu\}$ satisfying: (1) $\mu<\mu_{2}$; (2) $k(\mu)=3$; (3) there exists no $\mu^{*}$ with $k\left(\mu^{*}\right)>3$ such that $\mu<\mu^{*}$. From Hurwitz's theorem and the last statement of Theorem 7.5, it follows that $2 \leqq k\left(\mu_{3}\right) \leqq 3$. From Theorem 7.1, Hurwitz's theorem and the first statement of Theorem 7.5 it follows that $\left|\phi_{0}\left(x ; \mu_{3}\right)\right|$ attains $m\left(\mu_{3}\right)$ at least three times on $E_{r}$. Suppose now that $k\left(\mu_{3}\right)=2$. In this case $\left|\phi_{0}\left(x ; \mu_{3}\right)\right|$ would attain $m\left(\mu_{3}\right)$ precisely three times on $E_{r}$. But then the lesser of the two zeros of $\phi_{0}\left(z ; \mu_{8}\right)$ would necessarily exceed $-r$ and $\left|\phi_{0}\left(-r ; \mu_{3}\right)\right|=m\left(\mu_{3}\right)$, this last observation following from the fact that $\left|\phi_{0}\left(x ; \mu_{3}\right)\right|$ is monotone strictly decreasing for $x$ between -1 and the smallest $x$ on $E_{r}$ for which $\left|\phi_{0}\left(x ; \mu_{3}\right)\right|=m\left(\mu_{3}\right)$. Hence Lemma 7.2 implies that $\phi_{0}\left(z ; \mu_{3}\right) \equiv \pi_{2}(z)$. But Theorem 7.5 and Theorem 7.1 imply that $\phi_{0}\left(z ; \mu_{2}\right) \equiv \pi_{2}(z)$. The contradiction is manifest and it follows that $k\left(\mu_{3}\right)=3$. From a step-by-step argument modelled on these considerations it follows that there exists a strictly decreasing sequence $\left\{\mu_{\lambda}\right\}$ with $\mu_{0}=1, \mu_{1}=\rho$, and $\lim _{\lambda \rightarrow \infty} \mu_{\lambda}=0$, such that for $\mu_{\lambda} \leqq \mu<\mu_{\lambda-1}, k(\mu)=\lambda(\lambda=1,2, \cdots)$ and $\phi_{0}\left(z ; \mu_{\lambda}\right) \equiv \pi_{\lambda}(z)$. Hence of course $\left|\phi_{0}\left(x ; \mu_{\lambda}\right)\right|$ attains $m\left(\mu_{\lambda}\right)$ precisely $\lambda+1$ times for $x \in E_{r}$.

From this property of $\phi_{0}\left(z ; \mu_{\lambda}\right), \mu_{2}$ and $\phi_{0}\left(z ; \mu_{2}\right)$ can be calculated quite simply. Set

$$
\phi_{0}(z ; \mu) \equiv \frac{z-\alpha}{1-\alpha z} \cdot \frac{z-\beta}{1-\beta z}
$$


From $\phi_{0}\left(-r ; \mu_{2}\right)=\phi_{0}\left(r ; \mu_{2}\right)$ it follows that

$$
\frac{r+\alpha}{1+\alpha r} \cdot \frac{r+\beta}{1+\beta r}=\frac{r-\alpha}{1-\alpha r} \cdot \frac{r-\beta}{1-\beta r},
$$

and this implies $\alpha+\beta=0$. Hence $\phi_{0}\left(z ; \mu_{2}\right)$ may be written in the form

$$
\phi_{0}\left(z ; \mu_{2}\right) \equiv\left(z^{2}-\alpha^{2}\right) /\left(1-\alpha^{2} z^{2}\right) .
$$

Since the zeros are symmetrically located with respect to the origin, $\phi_{0}\left(0 ; \mu_{2}\right)$ must be a relative minimum and hence $\phi_{0}\left(0 ; \mu_{2}\right)=-\phi_{0}\left(r ; \mu_{2}\right)$, that is

$$
\left(r^{2}-\alpha^{2}\right) /\left(1-\alpha^{2} r^{2}\right)=\alpha^{2},
$$

whence

$$
\begin{aligned}
& \alpha^{2}=r^{2} /\left\{1+\left(1-r^{4}\right)^{1 / 2}\right\} ; \\
& \mu_{2}=\left\{\rho^{2}\left(1+\left(1-r^{4}\right)^{1 / 2}\right)-r^{2}\right\} /\left\{1+\left(1-r^{4}\right)^{1 / 2}-\rho^{2} r^{2}\right\} .
\end{aligned}
$$

8. Extensions of the results of $\S 7$. Let $E$ denote any set of points lying in $(-1<x<\rho)$ which is closed relative to this interval and has the property that l.u.b. $E<\rho$. An obvious extension of the problem treated in the last section is the following extremal problem:

To determine g.l.b. $\in_{\mathfrak{F}_{\mu}}\{$ l.u.b. $x \in E|\phi(x)|\}$ and the associated extremal functions.

Here, as throughout the present paper, the principal concern will be to discover descriptive properties of the extremal functions.

The case where g.l.b. $E>-1$ calls for no special attention except to remark that, when $E$ consists of only a finite number of points and $\mu$ is sufficiently small,

$$
\underset{\phi \in \mathfrak{O}_{\mu}}{\text { g.l.b. }}\left\{\max _{x \in E}|\phi(z)|\right\}=0,
$$

and there may be an infinite set of extremal functions. On the other hand, the case where $E$ is not compact relative to $(-1<x<\rho)$ is decidedly different, though it should be noted that the methods employed are much the same as those used in $\$ 7$, and this case will now be considered.

Let $\phi_{0}(z ; \mu, E)$ denote an extremal function of the present problem. It follows, as in $\$ 7$, from a proof patterned on that of Theorem 3.1 that the zeros of $\phi_{0}(z ; \mu, E)$ are real and lie strictly between -1 and 1.u.b. $E$; and from Lemma 7.2 with $\alpha_{2}=\beta_{2}$ that all the zeros are simple. Two cases present themselves: either the zeros of $\phi_{0}(z ; \mu, E)$ are finite in number, or else they are infinite in number. Suppose that the first case is at hand and let $\beta_{1}, \beta_{2}, \cdots, \beta_{N}$ denote these zeros with

$$
\beta_{1}>\beta_{2}>\cdots>\beta_{N}
$$


Clearly

$$
\prod_{k=1}^{N}\left(\frac{\rho-\beta_{k}}{1-\beta_{k} \rho}\right) \geqq \mu .
$$

Equality cannot occur in (8.2) because then

$$
\phi_{0}(z ; \mu, E) \equiv \prod_{k=1}^{N}\left(\frac{z-\beta_{k}}{1-\beta_{k} z}\right)
$$

and $\lim _{x \rightarrow-1}\left|\phi_{0}(x ; \mu, E)\right|=1$, which contradicts the hypothesis that g.l.b. $E$ $=-1$. Let $\mu^{*}$ denote the quotient of $\mu$ by the left member of (8.2) and consider the problem of determining the extremal function $\psi_{0}(z)$ which is to satisfy: (1) $\psi_{0}(z)$ is analytic and of modulus less than unity for $|z|<1 ;(2) \psi_{0}(z) \neq 0$ for $|z|<1$; (3) $\psi_{0}(\rho)=\mu^{*}$; (4) for a given $x$ with $-1<x<\rho,\left|\psi_{0}(x)\right|$ is less than or equal to $|\psi(x)|$ where $\psi(z)$ is any function satisfying the requirements (1), (2), (3) imposed on $\psi_{0}(z)$. It is readily verified that $\psi_{0}(z)$ is unique and is given by

$$
\psi_{0}(z) \equiv \exp \left\{\log \mu^{*} \frac{1-(z-\rho) /(1-\rho z)}{1+(z-\rho) /(1-\rho z)}\right\} \quad\left(\log \mu^{*} \text { real }\right)
$$

It is to be observed that $\psi_{0}(z)$ is independent of $x$.

Returning to the problem at hand note that then

$$
\phi^{*}(z) \equiv \psi_{0}(z) \prod_{k=1}^{N}\left(\frac{z-\beta_{k}}{1-\beta_{k} z}\right)
$$

would be extremal and that with $x>-1, \lim _{x \rightarrow-1} \phi^{*}(x)=0$. This would imply that $\phi^{*}(z)$ would attain

$$
\underset{\phi \in \mathfrak{B}_{\mu}}{\text { g.l.b. }}\{\underset{x \in E}{\{\text { l.u.b. }}|\phi(x)|\}
$$

on $E$ and only at a finite number of points. By recasting the argument of Theorem 2.2 to apply to the present situation and by noting that with $x>-1$, $\lim _{x \rightarrow-1} \phi^{*}(x)=0$, it follows that the assumption that $\phi_{0}(z ; \mu, E)$ has only a finite number of zeros is untenable.

Let $\left\{\beta_{k}\right\}(k=1,2, \cdots)$ with

$$
\beta_{1}>\beta_{2}>\cdots
$$$$
\left(\lim _{k \rightarrow \infty} \beta_{k}=-1\right)
$$

denote the zeros of the extremal function under consideration. A repetition of the argument just employed shows that $\phi_{0}(z ; \mu, E)$ is a Blaschke product $\left(^{8}\right)$ of the form

(8) For the theory of Blaschke products see chap. 4 of [6]. 


$$
\phi_{0}(z ; \mu, E)=\prod_{k=1}^{\infty} \frac{z-\beta_{k}}{1-\beta_{k} z} .
$$

No question concerning the convergence of this product without the customary constant factors arises since all the $\beta_{k}$ save a finite number of them are less than any real number which is greater than -1 .

The uniqueness of $\phi_{0}(z ; \mu, E)$ is now disposed of quite simply. Suppose that $\Phi_{0}$ is extremal and distinct from $\phi_{0}$; it follows that $\left(\phi_{0}+\Phi_{0}\right) / 2$ is also extremal. A known result $\left({ }^{9}\right)$ of the theory of Blaschke products states that a Blaschke product is analytic at every point of $|z|=1$ which is not a limit point of its zeros and has a modulus equal to one at such a point. Since $\left(\phi_{0}+\Phi_{0}\right) / 2$ must be a Blaschke product of the same type as $\phi_{0}$ and $\Phi_{0}$ (that is, with respect to the location of its zeros), it follows that for $z \neq-1,|z|=1$, $\phi_{0} \equiv \Phi_{0}$ and hence for all $z$ with $|z|<1, \phi_{0} \equiv \Phi_{0}$.

The uniqueness proof is complete.

Is the extreme value attained on $E$ ? This is to be answered in the affirmative, for otherwise an appropriate small displacement of two successive zeros subject to the restriction of Lemma 7.1 would imply the existence of an extremal function distinct from $\phi_{0}$. Further, it follows from this lemma that there are points of $E$ strictly between successive $\beta_{k}$ and that for at least one point of $E$ between successive $\beta_{k},\left|\phi_{0}\right|$ attains its extreme value. This has as a consequence that the relative maxima or minima between successive zeros are in magnitude at least equal to the extreme value. Hence to sum up we state the following theorem.

THEOREM 8.1. If $E$ consists of a finite number of points $\left\{x_{l}\right\}$ where $-1<x_{1}<x_{2}<\cdots<x_{n}<\rho$ and if $\mu<\prod_{k=1}^{n}\left(\rho-x_{k}\right) /\left(1-\rho x_{k}\right)$, then the problem has a trivial character. In all other cases the extremal function is unique.

If $E$ is compact, the extremal function is $a(1, k)$ ( $k$ depending upon the data) directly conformal mapping of $|z|<1$ onto itself.

If $E$ is not compact, then the extremal function is a Blasche product with its zeros all in the interval $(-1<x<1 . u . b . E),-1$ being their unique limit point. In addition, between successive zeros there are points of $E$ where $\left|\phi_{0}\right|$ attains its maximum on $E\left({ }^{10}\right)$.

\section{BiBLIOGRAPHY}

1. S. BeRnstein, Leçons sur les propriétés extrémales et la meilleure approximation des fonctions analytiques d'une variable réelle, Paris, 1926.

2. A. Beurling, Etudes sur un problème de majoration, Thesis, Upsala, 1933.

3. C. Carath todory, Conformal representation, Cambridge, 1932.

(9) Cf. [11] where this fact is noted.

(10) The case where $E$ is given by $-1<x \leqq r(<\rho)$ is noteworthy, for the extremal function can be calculated in terms of theta functions. It will be treated in detail in a forthcoming paper of the author. 
4. F. Carlson, Sur le module maximum d'une fonction analytique uniforme, Arkiv för matematik, astronomi och fysik vol. 26A, no. 9 (1938) pp. 1-13.

5. M. H. HeINs, Extremal problems for functions analytic and single-valued in a doublyconnected region, Amer. J. Math. vol. 62 (1940) pp. 91-106.

6. G. Julia, Principes géométriques d'analyse, part 1, Paris, 1932.

7. R. Nevanlinna, Eindeutige analytische Funktionen, Berlin, 1936.

8. - Ueber eine Minimumaufgabe in der Theorie der konformen Abbildung, Nachr. Ges. Wiss. Göttingen, 1933.

9. W. Rogosinski, Zum Schwarzschen Lemma, Jber. Deutschen Math. Verein. vol. 44 (1934) pp. 258-261.

10. E. Schmidt, Ueber den Millouxschen Satz, Preuss. Akad. Wiss. Sitzungsber., 1932.

11. W. SeIdel, On the cluster values of analytic functions, Trans. Amer. Math. Soc. vol. 34 (1932) pp. 1-21.

12. O. Teichmüller, Eine Verschärfung des Dreikreisesatzes, Deutsche Mathematik vol. 1 (1939) pp. 16-22.

13. CH. DE LA VAlleE Poussin, Leçons sur l'approximation des fonctions d'une variable réelle, Paris, 1919.

14. J. L. WALSH, Interpolation and approximation by rational functions in the complex domain, Amer. Math. Soc. Colloquium Publications, vol. 20, New York, 1935.

ILLINOIS INSTITUTE OF TEChNOLOGY 